\title{
Association study in naturally infected helminth layers shows evidence for influence of interferon-gamma gene variants on Ascaridia galli worm burden
}

\author{
Gesine Lühken ${ }^{1 *}$, Matthias Gauly ${ }^{2}$, Falko Kaufmann ${ }^{2}$ and Georg Erhardt ${ }^{1}$
}

\begin{abstract}
Single nucleotide polymorphisms (SNPs) in the genes for interleukin-4,-13 and interferon-gamma, and 21 additional SNPs which previously had been significantly associated with immune traits in the chicken, were genotyped in white and brown layer hens and analyzed for their association with helminth burden following natural infections. A nucleotide substitution located upstream of the promoter of the interferon-gamma gene was significantly associated with the log transformed number of Ascaridia galli in the brown layer line (genotype CC: $6.4 \pm 1.0$ worms; genotype CT: $11.7 \pm 2.2$ worms). Therefore, IFNG seems to be a promising candidate gene for further studies on helminth resistance in the chicken.
\end{abstract}

\section{Introduction, Methods, and Results}

In the European Community, animal welfare issues and changes in consumer demands have resulted in a ban of conventional cages for laying hens from 2012 on (Council Directive 1999/74). This has resulted in an increased importance of floor husbandry systems and consequently in a renewed relevance of helminthoses [1]. The development of drug resistance in nematodes $[2,3]$ and restrictions for the use of anthelmintics in food producing animals are two important aspects urging scientists to find alternative strategies for the control of gastrointestinal infections in laying hens. Estimated heritabilities and breed or line differences for immunological characteristics were not only shown in mammals but also in poultry $[4,5]$. Moreover, heritabilities estimated for parameters of susceptibility to helminthic infections, as mean worm or larvae counts [6-10], suggest that it is possible to select for helminth resistance in poultry.

Although immunity in birds is not as well understood as in mammals, it has been shown that as in mammals [11], helminth infection in chickens results in polarization

\footnotetext{
* Correspondence: Gesine.Luehken@agrar.uni-giessen.de 'Department of Animal Breeding and Genetics, Justus-Liebig University of Giessen, Ludwigstrasse 21B, 35390 Giessen, Germany

Full list of author information is available at the end of the article
}

towards a type 2 immune reaction, including augmented expression of interleukin-4 and interleukin-13 and diminished interferon-gamma expression [12]. In a single nucleotide polymorphism (SNP) study concerning innate and adaptive immune response across white and brown layer lines, 59 significant associations between immune traits and SNPs in immunological relevant genes were detected [13]; however, variants of interleukin-4 (IL4), interleukin-13 (IL13) and interferon-gamma (IFNG) genes were not included.

The aim of the present study was to determine genotypes of SNPs in the IL4, IL13 and IFNG genes and of 21 additional SNPs significantly associated with immune traits in white and brown commercial layer lines and to analyze their association with worm numbers resulting from a natural helminth infection in order to identify gene regions as promising candidates for further studies on parasite resistance in chickens.

Whole blood samples, numbers of adult worms of Ascaridia galli, Heterakis gallinarum, Capillaria spp. and tapeworms of 197 Lohmann Brown (LB) and 246 Lohmann Selected Leghorn (LSL) hens and pedigree data (sires) were available from a recent study conducted by Kaufmann et al. [6]. Briefly, in their experiment LB and LSL hens were reared under helminth-free conditions 
and kept afterwards together in a free range system. At the end of the laying period, hens were slaughtered and worms were counted according to the World Association for the Advancement of Veterinary Parasitology (WAAVP) guidelines. Whereas LB hens showed a significantly $(P<0.05)$ higher mean number of adult $\mathrm{H}$. gallinarum, Capillaria spp. and tapeworms compared to LSL animals, the latter had a tendency towards a higher number of adult $A$. galli worms. The estimated heritabilities for worm burdens of the different helminths and of the total worm burden ranged from 0.11 to 0.69 in LB and from 0.01 to 0.30 in LSL. Further details are given by Kaufmann et al. [6]. DNA was extracted from whole blood samples of these 443 hens using the Invisorb Blood Mini HTS 96 Kit (Invitek, Berlin, Germany). Quality and quantity of DNA were checked after extraction using a NanoDrop spectrophotometer (NanoDrop Technologies, Wilmington, USA).

In a previous work, we sequenced the 5 -flanking and all coding regions of IFNG, IL4 and IL13 in 20 chickens, 10 each from the white and brown layer White Leghorn and New Hampshire breeds (unpublished). Among the identified SNPs, only those which were polymorphic in at least one of the breeds were selected for genotyping. Preferably, they were located in or near functional gene regions. Three of the selected SNPs had not been listed in the database of genetic variation [14] and therefore sequence information for those was sent to GenBank [GenBank:HQ888866-HQ888868]. Genotyping of two IFNG and three IL4 SNPs was done by PCR restrictionfragment-length-polymorphism (RFLP) analysis. For this purpose forward and reverse primers for IFNG (SNP in 5'-flanking region: 5'-tgacccttaaccacatgatt-3' and 5'tcttaaagcatggtcctggaa-3', $194 \mathrm{bp}$; SNP in exon 4: 5'gcagttaagcctgagggatg-3' and 5'- cctcattcggtattttcaggtc-3', $462 \mathrm{bp}$ ) and for IL4 (SNPs in exon 1 and intron 1: 5'acctcacggggagagaaagt-3' and 5'-tcgagctggctttcctctta-3', 554 bp; SNP in intron 3: 5'-tgctgttctaatccactcaagaa-3' and 5'-aaagctgctcccatcttttc-3', $725 \mathrm{bp}$ ) were used to amplify DNA fragments that were digested with appropriate restriction enzymes (Table 1 ) according to the manufacturers' (MBI Fermentas, St. Leon-Rot, Germany; New England Biolabs, Frankfurt, Germany) recommendations. The last nucleotide of the forward primer for the IFNG 5'-flanking region was a mismatch in order to enable RFLP analysis by an amplification created restriction site [15].

SNP genotypes were discriminated after electrophoresis of the digested PCR products on agarose gels and ethidium bromide staining.

All other SNPs $(n=22)$ were genotyped with matrixassisted laser desorption/ionization time of flight mass spectrometry (MALDI-TOF MS) by Eurofins Medigenomix GmbH, Martinsried, Germany, using the Sequenom
Massarray iPLEX Gold System (Sequenom, San Diego, USA). Twenty-one SNPs were chosen from the study of Biscarini et al. [13]. All of these SNPs were significantly associated with at least one of the analyzed immune traits (production of natural antibodies against exo- and endoantigens and of acquired antibodies, activation of classical and alternative complement pathways) with a $P$-value < 0.01 [13]. Furthermore, a nucleotide substitution in intron 1 of IL13, already recorded in the db SNP database (rs15709642), was included in the MALDI-TOF MS multiplex assay.

For all genotyped SNPs, the rs number or a GenBank accession number, the chromosomal and gene location and the genotyping method used are given in Table 1.

SNP allele frequencies were calculated from the genotypes obtained. The significance of differences between allele frequencies of the genotyped SNPs in the two lines were analyzed with a chi square test, or with a Fisher exact test if the smallest cell contained less than six cases. Worm numbers were $\log$ transformed $[\log$ (worm number +10$)]$ to get approximately normally distributed data, as done before by Kaufmann et al. [6]. Association studies were performed for each SNP - showing a minor allele frequency $\geq 5 \%$ per line - with each of the observed parasitological traits, using the following statistical model: $\mathrm{y}_{i j}=\mu+\mathrm{SNP}_{i}+\mathrm{e}_{i j}$, where $y_{i j}$ represents the observation for the animal $j$, with SNP genotype $i$; $\mu$ is the overall mean of the trait; $\mathrm{SNP}_{i}$ is the effect of the SNP genotype, either $\mathrm{AA}, \mathrm{AB}$ or $\mathrm{BB}$; and $e_{i j}$ is the random residual effect. Association analysis was done within each line for all SNPs and additionally across lines for SNPs whose allele frequencies were not significantly $(P<0.05)$ different between the two lines.

Allele frequencies of the 27 genotyped SNPs are shown in Figure 1 for each line. A total of 14 SNPs was monomorphic and 1 additional SNP had a minor allele frequency $<0.05$ in LSL, whereas 3 SNPs were fixed and 3 showed a minor allele frequency $<0.05$ in LB. SNP 26, located in the $B L B 1$ region of the major histocompatibility complex $(M H C)$, was fixed in both lines. SNP 12 showed a minor allele frequency $<0.05$ in LB and was monomorphic in LSL. Therefore SNPs 12 and 26 were not included in association analyses in any of the two lines.

The SNPs in exon 1 and intron 1 of IL4 were genotyped by amplifying a single PCR product and digested with a single enzyme, enabling the demonstration of a total of 3 haplotypes (Figure 2). Only two of these haplotypes were identified in LB but all three in LSL (haplotype frequencies not shown).

Besides the fixed SNP 26, the allele frequencies of only two SNPs (7 and 21) were not significantly different between LB and LSL (Table 1). Therefore only those two SNPs were also analyzed for association with worm numbers across lines and not only within lines. 
Table 1 Numbers, locations, genotyping methods and P-values for allele frequency differences between lines of analyzed SNPs

\begin{tabular}{|c|c|c|c|c|c|}
\hline SNP no. & rs or GenBank accession no. & $\mathrm{GGA}^{1}$ & gene symbols (gene region) & $\begin{array}{l}\text { genotyping } \\
\text { method }^{2}\end{array}$ & $P$ (allele frequencies between lines) \\
\hline 1 & HQ888866 & 1 & IFNG (5'-flanking region) & PCR-RFLP (Hinfl) & $<0.001$ \\
\hline 2 & HQ888867 & 1 & IFNG (exon 4, synonymous) & PCR-RFLP (Mboll) & $<0.001$ \\
\hline 3 & rs13526054 & 3 & IL17F (exon, synonymous) & MALDI-TOF MS & $<0.001$ \\
\hline 4 & rs14082130 & 3 & MAL (intron) & MALDI-TOF MS & $<0.001$ \\
\hline 5 & rs15458146 & 3 & IL17F (exon, nonsynonymous) & MALDI-TOF MS & $<0.001$ \\
\hline 6 & rs13520872 & 4 & SHROOM3 (intron) & MALDI-TOF MS & $<0.001$ \\
\hline 7 & rs13520980 & 4 & NUP54 (intron) & MALDI-TOF MS & 0.834 \\
\hline 8 & rs13521841 & 4 & no gene & MALDI-TOF MS & $<0.001$ \\
\hline 9 & rs15475503 & 4 & HTR2C (intron) & MALDI-TOF MS & $<0.001$ \\
\hline 10 & rs13586560 & 5 & ENTPD5 (intron) & MALDI-TOF MS & $<0.001$ \\
\hline 11 & rs13586776 & 5 & FLVCR2 (intron) & MALDI-TOF MS & $<0.001$ \\
\hline 12 & rs13755931 & 5 & SPTBN5 (intron) & MALDI-TOF MS & 0.007 \\
\hline 13 & rs15669480 & 5 & TOLLIP (exon, synonymous) & MALDI-TOF MS & $<0.001$ \\
\hline 14 & rs14580491 & 6 & CXCL12 (intron) & MALDI-TOF MS & $<0.001$ \\
\hline 15 & rs13596817 & 7 & no gene & MALDI-TOF MS & $<0.001$ \\
\hline 16 & rs13596877 & 7 & no gene & MALDI-TOF MS & $<0.001$ \\
\hline 17 & rs13599559 & 7 & SPOPL (intron) & MALDI-TOF MS & $<0.001$ \\
\hline 18 & HQ888868 & 13 & IL4 (exon 1, synonymous) & PCR-RFLP (Taal) & $<0.001$ \\
\hline 19 & rs13505561 & 13 & IL4 (intron 1) & PCR-RFLP (Taal) & 0.012 \\
\hline 20 & rs15709667 & 13 & IL4 (intron 3) & PCR-RFLP (BCCl) & $<0.001$ \\
\hline 21 & rs14064765 & 13 & GMCSF (5'-flanking region) & MALDI-TOF MS & 0.248 \\
\hline 22 & rs14064896 & 13 & IRFI (3'-flanking region) & MALDI-TOF MS & $<0.001$ \\
\hline 23 & rs15677371 & 13 & no gene & MALDI-TOF MS & $<0.001$ \\
\hline 24 & rs15677377 & 13 & no gene & MALDI-TOF MS & $<0.001$ \\
\hline 25 & rs15709642 & 13 & IL13 (intron) & MALDI-TOF MS & $<0.001$ \\
\hline 26 & rs15788216 & 16 & $M H C, B L B 1$ (exon, nonsynonymous) & MALDI-TOF MS & 1.000 \\
\hline 27 & rs14119843 & 19 & HSPB1 (3'-flanking region) & MALDI-TOF MS & $<0.001$ \\
\hline
\end{tabular}

${ }^{1}$ number of chicken chromosome (Gallus gallus); ${ }^{2}$ for PCR-RFLP, used restriction enzymes are given in parentheses; CXCL12, chemokine (C-X-C motif) ligand 12; ENTPD5, ectonucleoside triphosphate diphosphohydrolase 5; FLVCR2, feline leukemia virus subgroup C cellular receptor family, member 2; GMCSF, granulocytemacrophage colony-stimulating factor; HSPB1, heat shock 27kDa protein 1; HTR2C, 5-hydroxytryptamine (serotonin) receptor 2C; IFNG, interferon gamma; IL4, interleukin 4; IL13, interleukin 13; IL17F; interleukin 17F; IRF1, interferon regulatory factor 1; $M A L$, mal, T-cell differentiation protein; $M H C$ (BLB1), major histocompatibility complex class II antigen B-F minor heavy chain; NUP54, nucleoporin 54kDa; SHROOM3, shroom family member 3; SPOPL, speckle-type POZ protein-like; SPTBN5, spectrin, beta, non-erythrocytic 5; TOLLIP, toll interacting protein.

$P$-values resulting from analysis of the association of SNP genotypes with worm numbers of A. galli, H. gallinarum, Capillaria spp., tapeworms and total worm burden are given in Table 2. Three SNPs (1 and 21, located in the 5'-flanking region of IFNG and GMCSF, respectively, and SNP 4, an intronic nucleotide substitution in $M A L)$ were significantly associated with one of the traits analyzed; whereas 7 SNPs showed a tendency towards significance for association with one or more of the traits (Table 2). Although only SNPs with a minor allele frequency $<5 \%$ were tested for association with the different parasitological traits, for some of the SNPs studied, the statistical significance or tendency towards significance of an association was obviously connected with a genotype only present in a small portion of hens $(<10 \%)$. This was also the case for the significant associations of SNPs 4 and 21 with parasitological traits.

For SNP 1, showing significant association with the log transformed worm number of $A$. galli in LB, genotype $C C$ was very frequent $(83 \%)$, whereas genotype $C T$ occurred in a lower frequency $(17 \%)$. The average A. galli worm number was $6.4 \pm 1.0$ in LB hens with the genotype $C C$, whereas it was $11.7 \pm 2.2$ in hens with the genotype $C T$. As 10 of the 19 LB sires had only progeny with the $C C$ genotype, the association analysis for SNP 1 regarding the number of $A$. galli in LB was repeated only with hens $(n=90)$ from the 9 other sires, resulting in a $P$-value of 0.011 . 


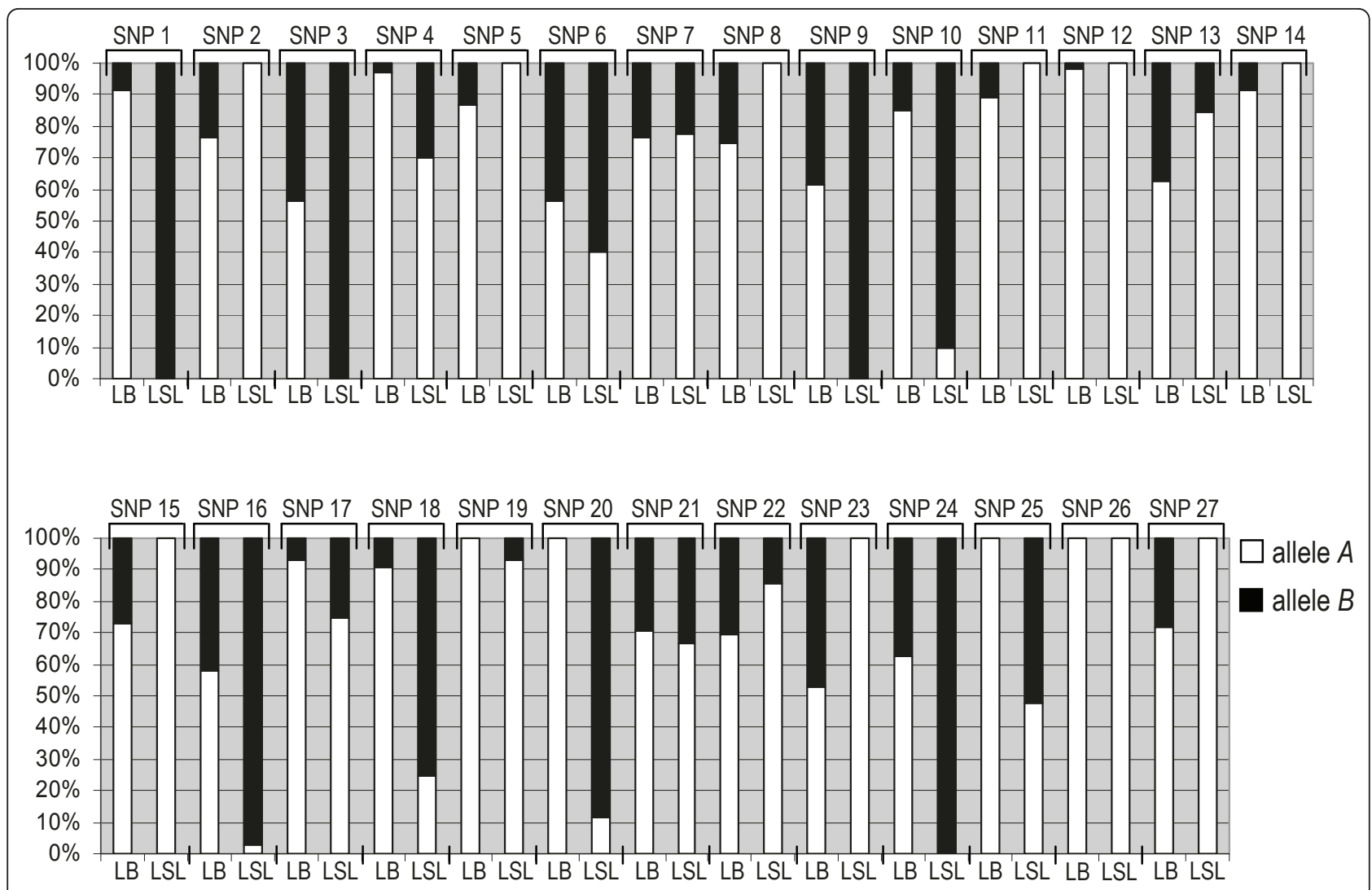

Figure 1 Allele frequencies (\%) of SNPs in Lohmann Brown (LB) and Lohmann Selected Leghorn (LSL) hens. For rs or GenBank accession numbers of SNPs and their location on chromosomes and in genes see Table 1.

\section{Discussion}

The higher number of monomorphic SNPs in the white layer line (52\% of the SNPs analyzed) compared to the brown (11\% of the SNPs analyzed) was in accordance with other studies $[13,16]$ and can be attributed to the smaller number of incorporated breeds in white lines $[16,17]$. Nevertheless, we did not expect such a large difference since Biscarini et al. [13] reported only $6 \%$ more fixed loci in 5 white layer lines compared to 4 in the brown lines. The larger difference observed in the present study could be due to the smaller number of analyzed SNPs or a higher homozygosity of LSL and/or lower homozygosity of LB compared to the average of the white and brown layer lines analyzed by Biscarini [13]. Interestingly, the lower homozygosity in LB is excluding the $A$. galli worm number- accompanied by higher heritabilities for worm numbers and at the same time significantly higher worm numbers, compared to LSL [6]. Among the SNPs which showed significant associations with parasitological traits, SNP 1 is the only one where this was not obviously linked to a very rare genotype. Genotypes $C C$ and $C T$ of SNP 1, a nucleotide substitution we previously identified in the IFNG 5'flanking region of New Hampshire and White Leghorn, were significantly associated with the number of $A$. galli worms in LB. In sheep, where nematode resistance has been a breeding goal much longer than in poultry, IFNG variants and markers located in the same chromosomal region as IFNG have already been associated with nematode resistance [18-20]. However, any of these polymorphisms were considered to directly influence the investigated trait. The chicken IFNG SNP analyzed here is located outside and upstream of the gene promoter [21]. Together with the monomorphic status of this SNP in LSL hens, showing a variance in A. galli worm numbers as in LB, it is more likely that its association with the A. galli number in LB is due to a linkage with a causal SNP in IFNG than influencing the worm number itself. Repeating the association analyses with other hens and with additional neighboring SNPs will be necessary to confirm the genetic influence of IFNG on susceptibility to A. galli in chickens that is supposed here. However, additional IFNG SNPs will be mainly located in non-coding gene regions, since the chicken 


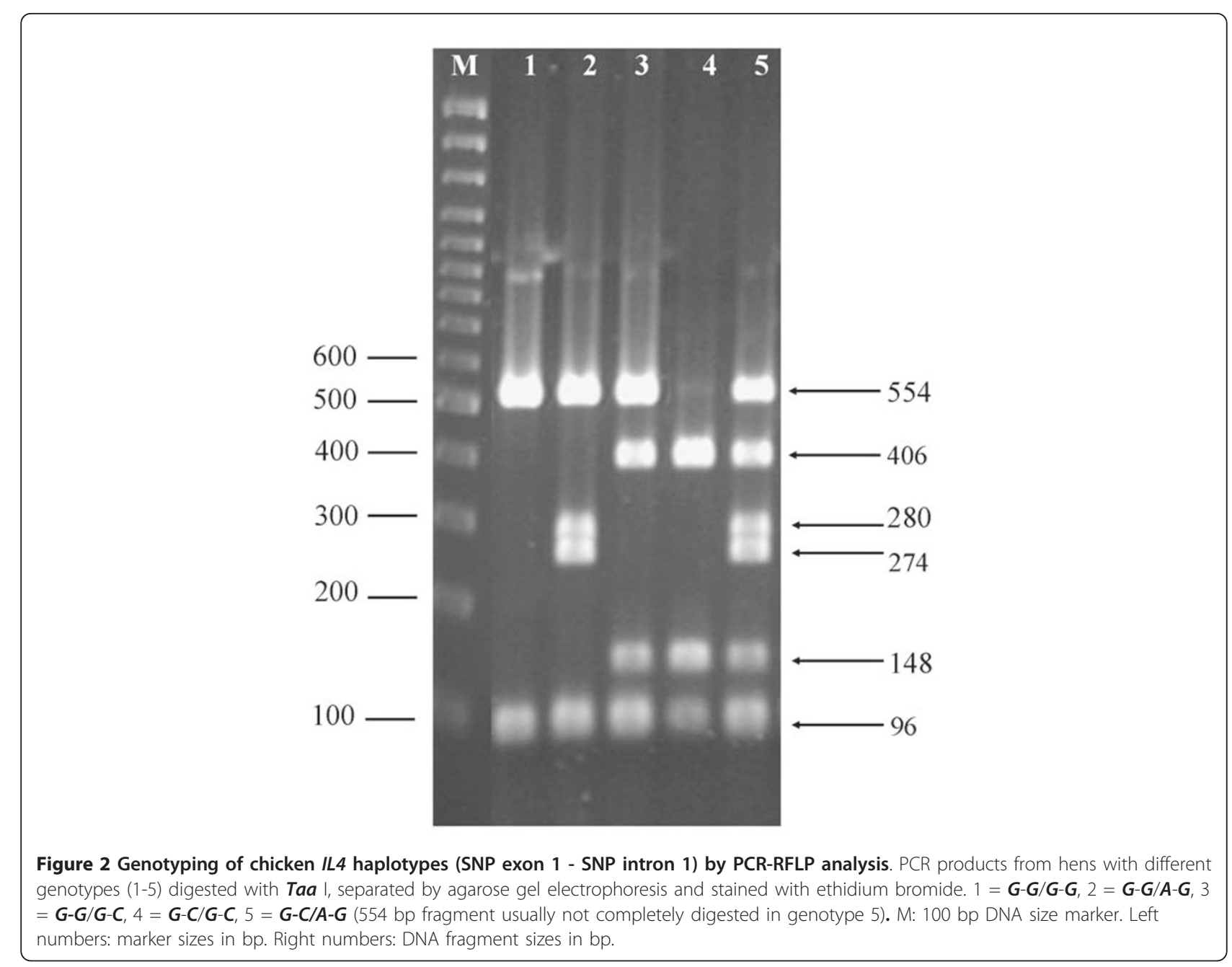

Table 2 P-values for association of SNPs with worm numbers in LB and LSL or both (all).

\begin{tabular}{|c|c|c|c|c|c|c|}
\hline SNP no. & line & A. galli & H. gallinarum & Capillaria ssp. & tapeworms & all helminths \\
\hline 1 & LB & 0.017 & 0.175 & 0.356 & 0.289 & 0.287 \\
\hline 2 & $\mathrm{LB}$ & 0.994 & 0.487 & 0.695 & 0.163 & 0.754 \\
\hline 3 & LB & 0.375 & 0.977 & 0.129 & 0.408 & 0.842 \\
\hline \multirow[t]{2}{*}{4} & $\mathrm{LB}$ & 0.479 & 0.052 & 0.077 & 0.215 & 0.133 \\
\hline & LSL & 0.186 & 0.472 & 0.540 & 0.992 & 0.280 \\
\hline 5 & $\mathrm{LB}$ & 0.301 & 0.983 & 0.152 & 0.494 & 0.855 \\
\hline \multirow[t]{2}{*}{6} & LB & 0.304 & 0.439 & 0.105 & 0.980 & 0.680 \\
\hline & LSL & 0.068 & 0.082 & 0.346 & 0.828 & 0.063 \\
\hline \multirow[t]{3}{*}{7} & $\mathrm{LB}$ & 0.838 & 0.892 & 0.913 & 0.757 & 0.916 \\
\hline & LSL & 0.793 & 0.231 & 0.604 & 0.537 & 0.265 \\
\hline & all & 0.984 & 0.673 & 0.659 & 0.886 & 0.716 \\
\hline 8 & LB & 0.493 & 0.203 & 0.722 & 0.479 & 0.153 \\
\hline 9 & LB & 0.086 & 0.515 & 0.154 & 0.693 & 0.316 \\
\hline \multirow[t]{2}{*}{10} & LB & 0.488 & 0.951 & 0.750 & 0.137 & 0.841 \\
\hline & LSL & 0.489 & 0.672 & 0.872 & 0.667 & 0.823 \\
\hline 11 & LB & 0.741 & 0.647 & 0.620 & 0.686 & 0.577 \\
\hline
\end{tabular}


Table 2 P-values for association of SNPs with worm numbers in LB and LSL or both (all). (Continued)

\begin{tabular}{|c|c|c|c|c|c|c|}
\hline 13 & LB & 0.687 & 0.215 & 0.926 & 0.743 & 0.165 \\
\hline & LSL & 0.205 & 0.398 & 0.067 & 0.600 & 0.212 \\
\hline 14 & LB & 0.535 & 0.306 & 0.365 & 0.734 & 0.487 \\
\hline 15 & LB & 0.677 & 0.225 & 0.669 & 0.639 & 0.219 \\
\hline \multirow[t]{2}{*}{16} & LB & 0.359 & 0.609 & 0.827 & 0.604 & 0.796 \\
\hline & LSL & 0.187 & 0.692 & 0.547 & 0.150 & 0.938 \\
\hline \multirow[t]{2}{*}{17} & LB & 0.500 & 0.126 & 0.141 & 0.216 & 0.319 \\
\hline & LSL & 0.081 & 0.375 & 0.146 & 0.537 & 0.179 \\
\hline 18 & LSL & 0.462 & 0.193 & 0.758 & 0.830 & 0.303 \\
\hline 19 & LSL & 0.613 & 0.317 & 0.676 & 0.620 & 0.427 \\
\hline 18-19 & LSL & 0.607 & 0.431 & 0.841 & 0.756 & 0.615 \\
\hline 20 & LSL & 0.815 & 0.521 & 0.828 & 0.847 & 0.517 \\
\hline \multirow[t]{3}{*}{21} & LB & 0.960 & 0.322 & 0.875 & 0.056 & 0.529 \\
\hline & LSL & 0.090 & 0.678 & 0.061 & 0.540 & 0.296 \\
\hline & all & 0.418 & 0.435 & 0.856 & 0.003 & 0.500 \\
\hline \multirow[t]{2}{*}{22} & LB & 0.628 & 0.292 & 0.371 & 0.878 & 0.459 \\
\hline & LSL & 0.605 & 0.182 & 0.337 & 0.789 & 0.200 \\
\hline 23 & LB & 0.067 & 0.213 & 0.138 & 0.411 & 0.122 \\
\hline 24 & LB & 0.930 & 0.938 & 0.327 & 0.207 & 0.623 \\
\hline \multirow[t]{2}{*}{25} & LB & 0.495 & 0.886 & 0.260 & 0.625 & 0.632 \\
\hline & LSL & 0.337 & 0.074 & 0.067 & 0.413 & 0.113 \\
\hline 27 & LB & 0.133 & 0.305 & 0.124 & 0.924 & 0.471 \\
\hline
\end{tabular}

Significant associations $(P<0.05)$ and associations with a tendency towards significance $(P<0.1)$ are bold typed.

IFNG is known for its high degree of sequence conservation especially in coding regions [21,22].

The existence of only weak linkage to a causal SNP may also be the reason that some SNPs only tended to be associated with one or more of the traits and in only one of the two lines. Therefore, additionally to SNPs in IFNG, some of them may be worth studying in further experiments, especially variants of IL13 as SNP 25 and other adjacent SNPs.

\section{Acknowledgements}

The animals originated from Lohmann Tierzucht GmbH, Cuxhaven, Germany. The authors thank the Ministry of Nutrition, Agriculture, Consumer Protection and State Development of Lower Saxony, Germany, for financial support.

\section{Author details}

${ }^{1}$ Department of Animal Breeding and Genetics, Justus-Liebig University of Giessen, Ludwigstrasse 21B, 35390 Giessen, Germany. ${ }^{2}$ Department of Animal Science, Georg-August University of Goettingen, Albrecht-Thaer-Weg 3, 37075 Goettingen, Germany.

\section{Authors' contributions}

GL conceived the study, designed and carried out the molecular genetic and association analyses and drafted the manuscript. MG conceived and designed the infection study, acquired funding and contributed to the interpretation of data. FK acquired, analyzed and interpreted the parasitological data. GE conceived the study, participated in its design and helped draft the manuscript. All authors read and approved the final manuscript.

\section{Competing interests}

The authors declare that they have no competing interests.

Received: 4 March 2011 Accepted: 12 July 2011 Published: 12 July 2011

\section{References}

1. Permin A, Bisgaard M, Frandsen F, Pearman M, Kold J, Nansen P: Prevalence of gastrointestinal helminths in different poultry production systems. Br Poult Sci 1999, 40:439-443.

2. Jackson F, Miller J: Alternative approaches to control-quo vadit? Vet Parasitol 2006, 139:371-384

3. Leignel V, Silvestre A, Humbert JF, Cabaret J: Alternation of anthelmintic treatments: a molecular evaluation for benzimidazole resistance in nematodes. Vet Parasitol 2010, 172:80-88.

4. Lamont SJ, Pinard MH, Cahaner A, van der Poel J, Parmentier HK: Selection for disease resistance: direct selection on the immune response. In Poultry Genetics, Breeding and Biotechnology. Edited by: Muir WM, Aggrey SE. Wallingford: CAB International; 2003:399-414.

5. Wijga S, Parmentier HK, Nieuwland MG, Bovenhuis H: Genetic parameters for levels of natural antibodies in chicken lines divergently selected for specific antibody response. Poult Sci 2009, 88:1805-1810.

6. Kaufmann F, Das G, Preisinger R, Schmutz M, König S, Gauly M: Genetic resistance to natural helminth infections in two chicken layer lines. Vet Parasitol 2011, 176:250-257.

7. Gauly M, Kanan A, Brandt H, Weigend S, Moors E, Erhardt G: Genetic resistance to Heterakis gallinarum in two chicken layer lines following a single dose infection. Vet Parasitol 2008, 155:74-79.

8. Gauly M, Bauer C, Preisinger R, Erhardt G: Genetic differences of Ascaridia galli egg output in laying hens following a single dose infection. Vet Parasitol 2002, 103:99-107.

9. Permin A, Ranvig $\mathrm{H}$ : Genetic resistance to Ascaridia galli infections in chickens. Vet Parasitol 2001, 102:101-111. 
10. Schou T, Permin A, Roepstorff A, Sorensen P, Kjaer J: Comparative genetic resistance to Ascaridia galli infections of 4 different commercial layerlines. Br Poult Sci 2003, 44:182-185.

11. Mosmann TR, Sad S: The expanding universe of T-cell subsets: Th1, Th2 and more. Immunol Today 1996, 17:138-146.

12. Degen WG, Daal N, Rothwell L, Kaiser P, Schijns VE: Th1/Th2 polarization by viral and helminth infection in birds. Vet Microbiol 2005, 105:163-167.

13. Biscarini F, Bovenhuis $H$, van Arendonk JA, Parmentier HK, Jungerius AP, van der Poel JJ: Across-line SNP association study of innate and adaptive immune response in laying hens. Anim Genet 2010, 41:26-38.

14. Database of genetic variation (dbSNP). [http://www.ncbi.nlm.nih.gov/snp].

15. Haliassos A, Chomel JC, Tesson L, Baudis M, Kruh J, Kaplan JC, Kitzis A: Modification of enzymatically amplified DNA for the detection of point mutations. Nucleic Acids Res 1989, 17:3606.

16. Hillel J, Groenen MA, Tixier-Boichard M, Korol AB, David L, Kirzhner VM, Burke T, Barre-Dirie A, Crooijmans RP, Elo K, Feldman MW, Freidlin PJ, MäkiTanila A, Oortwijn M, Thomson P, Vignal A, Wimmers K, Weigend S: Biodiversity of 52 chicken populations assessed by microsatellite typing of DNA pools. Genet Sel Evol 2003, 35:533-557.

17. Muir WM, Wong GK, Zhang Y, Wang J, Groenen MA, Crooijmans RP, Megens HJ, Zhang H, Okimoto R, Vereijken A, Jungerius A, Albers GA, Lawley CT, Delany ME, MacEachern S, Cheng HH: Genome-wide assessment of worldwide chicken SNP genetic diversity indicates significant absence of rare alleles in commercial breeds. Proc Natl Acad Sci USA 2008, 105:17312-17317.

18. Coltman DW, Wilson K, Pilkington JG, Stear MJ, Pemberton JM: A microsatellite polymorphism in the gamma interferon gene is associated with resistance to gastrointestinal nematodes in a naturally-parasitized population of Soay sheep. Parasitology 2001, 122:571-582.

19. Davies G, Stear MJ, Benothman M, Abuagob O, Kerr A, Mitchell S, Bishop SC: Quantitative trait loci associated with parasitic infection in Scottish blackface sheep. Heredity 2006, 96:252-258.

20. Sayers G, Good B, Hanrahan JP, Ryan M, Sweeney T: Intron 1 of the interferon gamma gene: Its role in nematode resistance in Suffolk and Texel sheep breeds. Res Vet Sci 2005, 79:191-196.

21. Kaiser $P$, Wain $H M$, Rothwell $L$ : Structure of the chicken interferon-gamma gene, and comparison to mammalian homologues. Gene 1998, 207:25-32.

22. Downing T, Lynn DJ, Connell S, Lloyd AT, Bhuiyan AK, Silva P, Naqvi AN, Sanfo R, Sow RS, Podisi B, O'Farrelly C, Hanotte O, Bradley DG: Contrasting evolution of diversity at two disease-associated chicken genes. Immunogenetics 2009, 61:303-314.

doi:10.1186/1297-9716-42-84

Cite this article as: Lühken et al:: Association study in naturally infected helminth layers shows evidence for influence of interferon-gamma gene variants on Ascaridia galli worm burden. Veterinary Research 2011 42:84.

\section{Submit your next manuscript to BioMed Central and take full advantage of:}

- Convenient online submission

- Thorough peer review

- No space constraints or color figure charges

- Immediate publication on acceptance

- Inclusion in PubMed, CAS, Scopus and Google Scholar

- Research which is freely available for redistribution 